\title{
Journal of Global Optimization Best Paper Award for a paper published in 2013
}

\author{
Sergiy Butenko
}

Received: 20 October 2014 / Accepted: 24 October 2014 / Published online: 30 October 2014

C) Springer Science+Business Media New York 2014

Journal of Global Optimization (JOGO) Best Paper Award, which consists of $\$ 1,000$ and a certificate, is awarded annually to a paper published in JOGO during the preceding year. The selection is made by a committee consisting of the Editor-in-Chief and several invited members of the editorial board. This year Immanuel Bomze, Mirjam Dür, Panos Pardalos, Oleg Prokopyev, and Mauricio G. C. Resende served on this important committee.

I am pleased to announce that the winner of the 2013 JOGO Best Paper Award is the following publication:

- Ruth Misener and Christodoulos A. Floudas, GloMIQO: Global mixed-integer quadratic optimizer (volume 57, pages 3-50).

The abstract of the article reads:

This paper introduces the global mixed-integer quadratic optimizer, GloMIQO, a numerical solver addressing mixed-integer quadratically-constrained quadratic programs to $\epsilon$-global optimality. The algorithmic components are presented for: reformulating user input, detecting special structure including convexity and edgeconcavity, generating tight convex relaxations, partitioning the search space, bounding the variables, and finding good feasible solutions. To demonstrate the capacity of GloMIQO, we extensively tested its performance on a test suite of 399 problems of diverse size and structure. The test cases are taken from process networks applications, computational geometry problems, GLOBALLib, MINLPLib, and the Bonmin test set. We compare the performance of GloMIQO with respect to four state-of-the-art global optimization solvers: BARON 10.1.2, Couenne 0.4, LindoGLOBAL 6.1.1.588, and SCIP 2.1.0.

According to the authors,

"The computational framework described in this manuscript was released as solver software by the GAMS Development Corporation (GloMIQO; first available in GAMS

S. Butenko $(\varangle)$

Texas A\&M University, College Station, TX, USA

e-mail: butenko@tamu.edu 
23.8) and has been used for diverse research applications such as: optimal crystal orientation; multiperiod blend scheduling; network flow; water network design; cutting ellipses from area-minimizing rectangles; gasoline blend planning by researchers from: BASF; Carnegie Mellon University; ExxonMobil; Imperial College London; Laboratorio Nacional de Energia e Geologia (Portugal); McMaster University; Princeton University; University of Florida; etc. GloMIQO continues to be under active development and is now available in a second version [1]. From a more theoretical/computational point of view, GloMIQO has been used as a point of comparison for new algorithms including: multiparametric disaggregation for bilinear programs; linear cutting planes for network flow problems; heuristic strategies. The GloMIQO framework has also been extended to incorporate Mixed-Integer Signomial Optimization (MISO) and Mixed-Integer Nonlinear Programming (MINLP) problems via the algorithms in ANTIGONE [2,3]; first available in GAMS 24.1."

In addition, the following articles were recognized as "Top-5 Papers Published in JOGO in 2013" (listed in order of their appearance in the published issues):

- Lewis Ntaimo, Fenchel decomposition for stochastic mixed-integer programming (volume 55, pages 141-163).

- Luis Miguel Rios and Nikolaos V. Sahinidis, Derivative-free optimization: a review of algorithms and comparison of software implementations (volume 56, pages 1247-1293).

- Agustín Bompadre, Alexander Mitsos, and Benoît Chachuat, Convergence analysis of Taylor models and McCormick-Taylor models (volume 57, pages 75-114).

- Constantin Zălinescu, On the differentiability of the support function (volume 57, pages 719-731).

Congratulations to the winners, and thanks to Springer and everyone involved in the committee and nomination process for their support. I would also like to use this opportunity to invite nominations for the 2014 JOGO Best Paper Award. All JOGO papers published in 2014 (volumes 58-60) are eligible. Please email your nominations to butenko@ tamu.edu.

\section{References}

1. http://helios.princeton.edu/GloMIQO/index.html

2. Misener, R., Floudas, C.A.: A framework for globally optimizing mixed-integer signomial programs. J. Optim. Theory Appl. 161, 905-932 (2014)

3. Misener, R., Floudas, C.A.: ANTIGONE: algorithms for coNTinuous/integer global optimization of nonlinear equations. J. Glob. Optim. 59, 503-526 (2014) 